Ann. Sci. forest., 1972, 29 (4), 451-463.

\title{
INFLUENCE DE LA DATE ET DE LA HAUTEUR DU PRÉLÈVEMENT SUR LES RÉSULTATS DE L'ANALYSE FOLIAIRE CHEZ DEUX CLONES DE PEUPLIER
}

\author{
J. GARBAYE \\ avec la collaboration technique de G. Courriér \\ Station de Recherches sur les Sols Forestiers et la Fertilisation, \\ Centre national de Recherches forestières, I.N.R.A. \\ Champenoux 54370 Einville
}

\begin{abstract}
RÉSUMÉ
Dans le but de déterminer la meilleure façon de prélever les feuilles en vue du diagnostic foliaire chez le Peuplier, des prélèvements mensuels ont été faits à trois hauteurs différentes sur deux peupleraies de la vallée de la Marne, l'une plantée en $P$. $x$ euramericana $\mathrm{cv}$ 《 Robusta », l'autre en $P . x$ euramericana $\mathrm{cv} \ll \mathrm{I}-214 »\left({ }^{1}\right)$.

L'interprétation statistique des résultats entraîne les conclusions suivantes :

- les feuilles du sommet de l'arbre sont les plus intéressantes pour le diagnostic foliaire du fait de leur forte teneur en macroéléments et de leur activité métabolique plus intense qui donne une meilleure estimation de l'état nutritionnel de l'arbre;

- le prélèvement doit avoir lieu fin août ou début septembre car la composition des feuilles varie peu pendant cette période;

- les deux clones ont des compositions foliaires différentes; par conséquent, le diagnostic foliaire doit être fondé sur des valeurs critiques propres à chaque clone;

- la comparaison des résultats obtenus en 1968 et 1969 sur les mêmes arbres montre de grandes différences. Au cours d'une enquête, il est donc impossible de comparer les résultats d'analyses foliaires faites sur plusieurs années ou dans des régions trop différentes du point de vue climatique.
\end{abstract}

\section{INTRODUCTION}

Avant d'entreprendre une enquête sur la production et la nutrition minérale du peuplier dans le Bassin Parisien, nous avons été amenés à définir les conditions optimales d'époque et de hauteur de prélèvement en vue du diagnostic foliaire.

(1) Désignés dans la suite de l'article par : Robusta et I-214. 
De nombreux auteurs ont déjà abordé ce problème. Citons par exemple VAN DER Meiden (1959) qui sur des Robusta de 9 ans trouve que le niveau de prélèvement n'a pas d'influence sur les teneurs en $\mathrm{N}, \mathrm{P}, \mathrm{K}$ et $\mathrm{Mg}$. Cependant, la teneur en Ca décroîtrait vers le haut de l'arbre.

Une étude anonyme sur Robusta et Gelrica (WAGENINGEN, 1961) confirme cette constance, étendue, cette fois, au calcium.

Cependant, en 1968, nous avons fait des observations contraires sur des Robusta de 12 ans dans la vallée de la Marne : N, P, et K étaient moins abondants dans les feuilles du bas de l'arbre, et Ca présentait la répartition inverse.

En ce qui concerne les variations saisonnières, le désaccord est aussi complet : pour VAN DER Meiden et Kolster (1959), la concentration diminue tout au long de la saison de végétation pour $\mathrm{N}$ et $\mathrm{P}$, reste constante pour $\mathrm{K}$ et $\mathrm{Mg}$, et présente un maximum en août pour Ca.

Schmalfuss et Schulze (1961) trouvent des résultats différents : maximum en août pour $\mathrm{N}$, décroissance continue pour $\mathrm{P}$, et augmentation continue pour $\mathrm{K}, \mathrm{Ca}$ et $\mathrm{Mg}$.

L'étude anonyme de WAGENINGEN (1961) signale un maximum en août pour $\mathrm{N}$ et $\mathrm{P}$, une augmentation pour $\mathrm{Ca}$ et la constance pour $\mathrm{K}$ et $\mathrm{Mg}$.

Les résultats provenant des diverses sources ne concordent pas, mais il ne faut pas oublier que ces travaux ont été faits sur des clones différents et dans des régions différentes.

Devant de telles variations au cours de la saison de végétation, Schmalfuss et SCHulze (1961) concluent que le diagnostic foliaire ne peut être que de peu de valeur pour les feuillus en général et le peuplier en particulier. Cependant, d'après l'ensemble des études citées, la période la moins mauvaise (où les variations sont les plus faibles) semble se situer fin août ou début septembre, ce qui correspond à ce que Leroy (1968) a observé sur le chêne dans l'Est de la France : deuxième quinzaine d'août.

Pour la France, Touzet, Heinrich et Nohn (1969) et Touzet et Heinrich (1970) trouvent une diminution régulière pour $\mathrm{N}, \mathrm{P}$ et $\mathrm{K}$. Par contre, pour le $\mathrm{Ca}$, ils constatent une différence entre le Bassin Parisien (augmentation continue) et la vallée de l'Isère (maximum en juin-juillet).

Devant ces imprécisions et contradictions, il nous a donc semblé utile d'entreprendre une recherche permettant de nous faire une opinion personnelle. Rappelons que le choix d'une période optimale et d'un niveau de prélèvement devrait répondre à plusieurs préoccupations.

- assurer, dans chaque station, une aussi faible dispersion que possible des résultats entre arbres.

- assurer le maximum de différenciation entre les stations à étudier.

- assurer une faible variation de la composition foliaire dans le temps. En effet, les études de relations sol-production que nous devons entreprendre supposent chaque année, sur un grand nombre de stations, parfois assez distantes les unes des autres, un prélèvement de matériel foliaire qui peut demander plusieurs semaines. Si nous faisons nos prélèvements pendant une période de rapide variation de concentration des éléments minéraux dans la feuille, la comparaison entre stations serait biaisée.

Parmi ces préoccupations, les deux dernières sont de loin, dans le travail entrepris, les plus importantes. 


\section{I. - MATÉRIEL ET MÉTHODES}

En 1969 , notre étude a porté sur une plantation de Robusta de 12 ans et sur une plantation de I-214 de 7 ans, toutes deux sur sol alluvial calcaire et à environ $500 \mathrm{~m}$ de distance pour éviter de trop grandes différences édaphiques ou climatiques (Vallée de la Marne, près de Vitry-le-François).

Ces plantations présentent une très bonne croissance; nous sommes donc assurés de trouver les valeurs de caractères foliaires au voisinage de leur optimum.

Dans chaque plantation, cinq arbres voisins ont fait l'objet de notre étude. Le tableau 1 donne les caractéristiques de ces arbres.

TABLEAU 1 - TABLE 1

Caractéristiques des arbres étudiés

Characteristics of trees

\begin{tabular}{|c|c|c|c|c|c|c|c|c|c|c|}
\hline Clone & \multicolumn{5}{|c|}{ Robusta } & \multicolumn{5}{|c|}{$1-214$} \\
\hline Age de la plantation & \multicolumn{5}{|c|}{12 ans } & \multicolumn{5}{|c|}{7 ans } \\
\hline $\mathrm{n}^{0}$ des arbres $\ldots \ldots \ldots \ldots$ & 1 & 2 & 3 & 4 & 5 & 1 & 2 & 3 & 4 & 5 \\
\hline circonférence à $1,30 \mathrm{~m}(\mathrm{~cm})$. & 76 & 82 & 73 & 78 & 78 & 63 & 60 & 60 & 57 & 62 \\
\hline hauteur $(\mathrm{m})$ & 18,5 & 17,5 & 18 & 20 & 20 & 12,1 & 13,5 & 12,9 & $' 12,7$ & 13,3 \\
\hline
\end{tabular}

Les prélèvements ont été effectués sur les 10 arbres à cinq dates différentes ( 2 juin, 9 juillet, 4 août, 8 septembre et 7 octobre) et 3 niveaux : tiers supérieur du feuillage $(\mathrm{H})$, tiers médian $(\mathrm{M})$ et tiers inférieur (B). La technique de prélèvement est la suivante :

A chaque niveau, quatre branches dans la direction des quatre points cardinaux sont coupées au fusil (calibre 12, chevrotines 28 grains). Cet échantillonnage autour de l'arbre a pour but d'éliminer d'éventuelles variations de composition des feuilles dues à l'exposition.

Sur chaque branche, les feuilles de la partie médiane des rameaux longs sont cueillies. Dans le cas des branches basses, les rameaux longs sont rares et représentés presque uniquement par les pousses terminales des branches.

Les feuilles de 4 branches d'un même niveau sont réunies et constituent un échantillon unique de 50 à 100 feuilles environ.

Cette manière d'opérer présente des avantages et des inconvénients : il serait préférable de situer les périodes de prélèvement par rapport à des «stades physiologiques repères », comme cela se fait pour les arbres fruitiers. Mais nous cherchons avant tout, rappelons-le, à mettre au point une méthode de diagnostic foliaire applicable à une enquête sur un grand nombre de stations. Pour des raisons de temps et de contraintes matérielles, nous sommes donc amenés à chercher la simplicité dans la technique de prélèvement. Pour la même raison, nous n'avons pas pris les feuilles en un endroit bien précis des rameaux, mais nous avons simplement éliminé les feuilles de la base de la pousse (souvent endommagées et parasitées) et les feuilles de l'extrémité juste formées et d'apparence juvénile (couleur, consistance). Nous n'avons donc pas tenu compte des différences pouvant exister entre feuilles 
préformées dans le bourgeon (pousse de printemps) et feuilles estivales. De plus, il convient de noter que les feuilles prélevées n'ont pas le même stade de développement à chaque date puisque le rameau s'allonge et que de nouvelles feuilles apparaissent.

Sur chaque échantillon, les caractères suivants ont été déterminés :

$1^{\circ}$ Caractères physiques et morphologiques (sur un échantillon de 50 feuilles) :

- L : longueur moyenne du limbe

$-\mathrm{I}_{a}:$ indice d'allongement $=\frac{\mathrm{L}}{\text { largeur moyenne }}$

- $\mathbf{P}_{s}$ : poids sec de 100 feuilles (en grammes) avec le pétiole.

$-\mathbf{I}_{d}:$ indice de densité $=\frac{\mathrm{P}_{s}}{\mathrm{~S}}\left(\mathrm{en} \mathrm{mg} / \mathrm{cm}^{2}\right) ; \mathrm{S}$ étant un indice de surface $: \mathrm{S}=\mathrm{L} \times$ largeur moyenne.

$2^{\circ}$ Teneur en eau (exprimée en pourcent de matière sèche) par séchage à l'étuve à $65^{\circ} \mathrm{C}$ pendant $24 \mathrm{~h}$ des feuilles avec le pétiole.

$3^{\circ}$ Teneurs en éléments minéraux totaux (exprimées en pourcent de matière sèche à $65^{\circ} \mathrm{C}$ pendant 24 heures, pétiole compris) :

- N p. cent : minéralisation par $\mathrm{H}_{2} \mathrm{SO}_{2}$ et $\mathrm{H}_{2} \mathrm{O}_{4}$; dosage par distillation Kjeldahl.

$-\mathrm{P}_{2} \mathrm{O}_{5}$ p. cent : minéralisation par $\mathrm{HClO}_{4}$ et $\mathrm{H}_{2} \mathrm{O}_{2}$; dosage par colorimétrie (réactif sulfomolybdique avec chlorure stanneux comme réducteur).

- K p. cent et Ca p. cent : minéralisation par $\mathrm{HClO}_{4}$ et $\mathrm{H}_{2} \mathrm{O}_{2}$; dosage par photométrie de flamme (émission).

- $\mathrm{Mg}$ p. cent : minéralisation par $\mathrm{HClO}_{4}$ et $\mathrm{H}_{2} \mathrm{O}_{2}$; dosage par colorimétrie au jaune de titane.

Enfin nous avons calculé $\mathrm{N}(100), \mathrm{P}_{2} \mathrm{O}_{5}(100) \ldots \mathrm{Mg}(100)$ qui sont les quantités totales (en grammes) de $\mathrm{N}, \mathrm{P}_{2} \mathrm{O}_{5} \ldots \mathrm{Mg}$ contenues dans 100 feuilles.

Nous n'avons pas tenu compte de résultats concernant le niveau supérieur à la date du 4 août chez les Robusta : les branches cassaient mal et n'ont pas permis la constitution d'un échantillon convenable.

\section{II. - RÉSULTATS}

Les figures 1 et 2 présentent les résultats obtenus sous forme graphique :

Pour chaque caractère foliaire, trois graphiques correspondent aux trois niveaux $\mathrm{H}, \mathrm{M}$ et $\mathrm{B}$.

En abscisse, sont portées les dates $\left(D_{1}, D_{2}, D_{3}, D_{4}, D_{5}\right)$ et en ordonnée, les valeurs du caractère considéré.

Pour chaque clone, une analyse de la variance a été faite entre dates, à chaque niveau, et entre niveau à chaque date (les cinq arbres constituent cinq répétitions). Les résultats de cette analyse statistique sont portés sur le même graphique :

Une matrice de 15 points représente les dates ( 5 colonnes) et les niveaux de prélèvement (3 lignes). Un astérisque en face d'une ligne ou d'une colonne indique que cette ligne ou cette colonne présente une différence globale significative au seuil de $5 \mathrm{p}$. cent entre ses points.

Enfin, les points qui ne diffèrent pas significativement entre eux au seuil de $5 \mathrm{p}$. cent, sont reliés par un trait. 

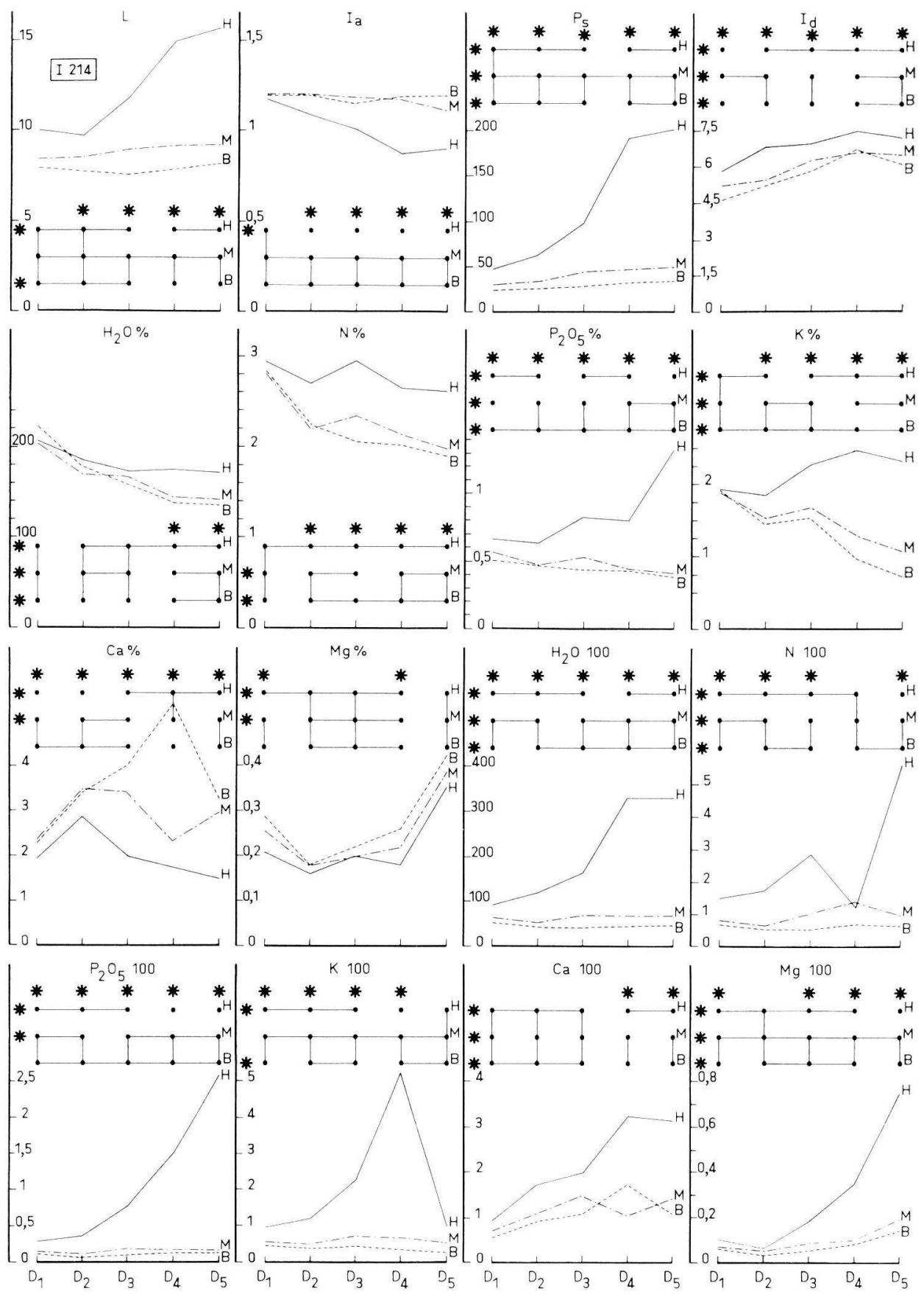

FIG. 1. - Variation des caractères foliaires en fonction de la date et du niveau de prélèvement (1-214) Influence of date and level of sampling on foliar characteristics (1-214) 

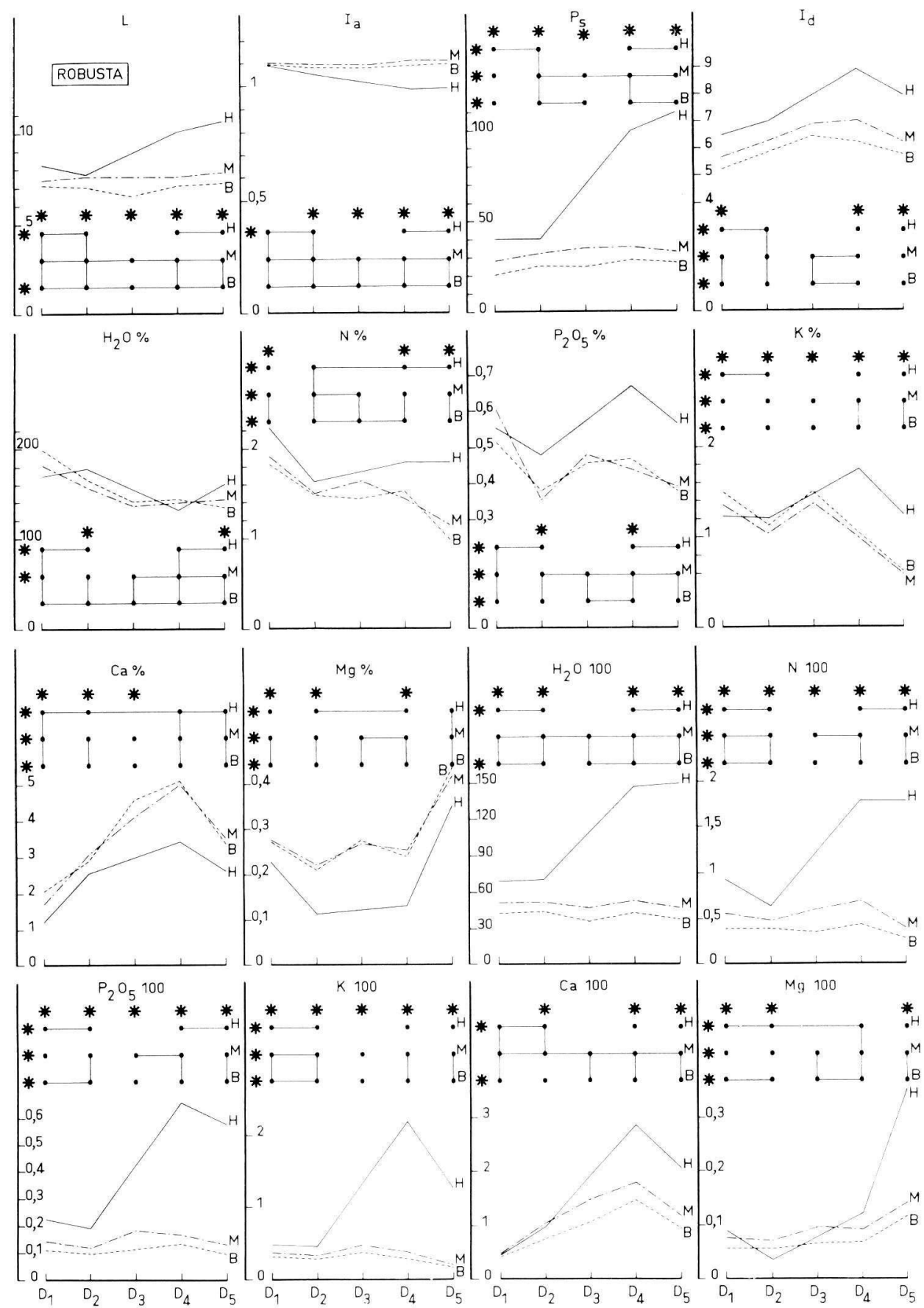

FIG. 2. - Variation des caractères foliaires en fonction de la date et du niveau de prélèvement (Robusta) Influence of date and level of sampling on foliar characteristics (Robusta) 
Le tableau 2 compare les valeurs observées le 8 septembre sur les feuilles hautes et les valeurs considérées comme optimales.

TABLEAU 2 - TABLE 2

Comparaison des valeurs observées le 8 septembre sur les feuilles hautes et des valeurs considérées comme optimales

Comparaison between mineral content of top leaves values measured on september $8 \mathrm{th}$, and optimum values

\begin{tabular}{|c|c|c|c|c|c|}
\hline Élément & $N$ p. cent & $\mathrm{P}_{2} \mathrm{O}_{5}$ p. cent & K p. cent & Ca p. cent & Mg p. cent \\
\hline Robusta $\ldots \ldots \ldots \ldots$ & 1,85 & 0,67 & 1,75 & 3,40 & 0,19 \\
\hline $1-214 \ldots \ldots \ldots \ldots \ldots \ldots$ & 2,65 & 0,80 & 2,5 & 1,80 & 0,18 \\
\hline Valeurs optimales ...... & 2,2 & 0,4 à 0,7 & 1,2 à 1,5 & $?$ & 0,15 \\
\hline
\end{tabular}

Le tableau 3 donne les caractères des feuilles tombées à terre sous les Robusta au mois d'octobre, et le tableau 4 permet de comparer les caractères foliaires entre deux années consécutives pour des dates voisines.

\section{III. - DISCUSSION}

\section{1. - Influence du niveau de prélèvement}

On remarque sur les figures 1 et 2 que les niveaux $M$ et $B$ présentent des valeurs très voisines et souvent non significativement différentes. Par contre, le niveau $\mathrm{H}$ s'individualise nettement. Les valeurs observées en $\mathbf{H}$ sont les plus fortes pour tous les caractères, sauf pour $\mathrm{H}_{2} \mathrm{O}$ p. cent, Ia (les feuilles hautes croissent plus vite en largeur qu'en longueur), Ca p. cent et $\mathrm{Mg}$ p. cent.

En ce qui concerne le calcium et le magnésium, le même phénomène a été observé par VAN Der MeIden et Kolster en 1964 sur des arbres de 9 ans. Cependant ces auteurs ne trouvaient aucune influence du niveau de prélèvement pour les autres caractères.

Nos résultats peuvent s'expliquer de la façon suivante, compte tenu de notre méthode de prélèvement :

La croissance des pousses dans les parties basses et moyennes de l'arbre s'arrête de bonne heure (juin). Il n'y a plus de feuilles nouvelles à partir de juillet, d'où la constance de certains caractères. Par contre, les pousses du haut de l'arbre continuent à former de nouvelles feuilles jusqu'au début de septembre pour Robusta ou mi-septembre pour I-214. Cette activité prolongée du haut de l'arbre amène à penser que les feuilles hautes, plus exposées à la lumière, ont une activité métabolique supérieure à celle des feuilles moyennes ou basses, et que par conséquent elles traduisent mieux les conditions de nutrition minérale de l'arbre. 
Caractères des feuilles tombées à terre le 7-10-69 (Robusta)

comparés à ceux des feuilles restées sur les arbres

Results of measurements on fallen leaves on october 7 th 1969 (Robusta) compared with those of leaves still on trees

\begin{tabular}{|c|c|c|c|c|c|c|c|c|c|c|c|c|c|c|c|c|}
\hline Niveau & $\underset{\mathrm{cm}}{\mathrm{L}}$ & Ia & $\begin{array}{c}\text { Ps } \\
\mathrm{g}\end{array}$ & $\underset{\mathrm{Id}}{\mathrm{Id} / \mathrm{cm}_{2}}$ & $\begin{array}{c}\mathrm{H}_{2} \mathrm{O} \\
\text { p. cent }\end{array}$ & p. cent & $\begin{array}{c}\mathrm{P}_{2} \mathrm{O}_{5} \\
\text { p. cent }\end{array}$ & $\begin{array}{l}\mathrm{K} \\
\text { p. cent }\end{array}$ & $\begin{array}{c}\mathrm{Ca} \\
\text { p. cent }\end{array}$ & $\begin{array}{c}\mathrm{Mg} \\
\text { p. cent }\end{array}$ & $\begin{array}{l}\mathrm{H}_{2} \mathrm{O} \\
(100)\end{array}$ & $\underset{(100)}{N}$ & $\begin{array}{l}\mathrm{P}_{2} \mathrm{O}_{5} \\
(100)\end{array}$ & $\begin{array}{c}\mathrm{K} \\
(100)\end{array}$ & $\begin{array}{c}\mathrm{Ca} \\
(100)\end{array}$ & $\begin{array}{c}\mathrm{Mg} \\
(100)\end{array}$ \\
\hline $\begin{array}{c}\mathrm{H} \\
\mathrm{M} \\
\mathrm{B}\end{array}$ & $\begin{array}{r}10,6 \\
7,8 \\
7,3\end{array}$ & $\begin{array}{l}1,00 \\
1,10 \\
1,12\end{array}$ & $\begin{array}{r}110,0 \\
34,0 \\
27,5\end{array}$ & $\begin{array}{l}7,99 \\
6,30 \\
5,70\end{array}$ & $\begin{array}{l}160,0 \\
143,5 \\
136,4\end{array}$ & $\begin{array}{l}1,85 \\
1,15 \\
1,00\end{array}$ & $\begin{array}{l}0,73 \\
0,39 \\
0,38\end{array}$ & $\begin{array}{l}1,24 \\
0,60 \\
0,63\end{array}$ & $\begin{array}{l}2,62 \\
3,40 \\
3,38\end{array}$ & $\begin{array}{l}0,35 \\
0,41 \\
0,43\end{array}$ & $\begin{array}{r}150 \\
46 \\
40\end{array}$ & $\begin{array}{l}1,78 \\
0,38 \\
0,28\end{array}$ & $\begin{array}{l}0,57 \\
0,13 \\
0,10\end{array}$ & $\begin{array}{l}1,25 \\
0,25 \\
0,20\end{array}$ & $\begin{array}{l}2,05 \\
1,20 \\
0,90\end{array}$ & $\begin{array}{l}0,35 \\
0,14 \\
0,12\end{array}$ \\
\hline Feuilles tombées $\ldots$. & 7,86 & 1,09 & 36,7 & 6,49 & 143,8 & 0,92 & 0,36 & 0,76 & 3,20 & 0,40 & 53 & 0,34 & 0,13 & 0,28 & 1,17 & 1,145 \\
\hline
\end{tabular}

TABLEAU 4 - TABLE 4

Comparaison des caractères foliaires entre le 24-8-68 et le 8-9-69

(moyenne des cinq arbres étudiés - feuilles du bas).

Comparison of results of measurement on leaves between august 24th, 1968 and september 8th, 1969 (mean values for the 5 trees - bottom leaves)

\begin{tabular}{|c|c|c|c|c|c|c|c|c|c|c|c|c|c|c|c|c|c|}
\hline Clone & Année & $\underset{\mathrm{cm}}{\mathrm{L}}$ & Ia & $\begin{array}{c}\text { Ps } \\
\mathrm{g}\end{array}$ & $\underset{\mathrm{mg} / \mathrm{cm}^{2}}{\mathrm{Id}}$ & $\mid \begin{array}{c}\mathrm{H}_{2} \mathrm{O} \\
\text { p. cent }\end{array}$ & $\stackrel{\mathrm{N}}{\text { p. cent }}$ & $\mid$\begin{tabular}{|c|}
$\mathrm{P}_{2} \mathrm{O}_{5}$ \\
p. cent
\end{tabular} & $\begin{array}{c}\mathrm{K} \\
\text { p. cent }\end{array}$ & $\begin{array}{c}\text { Ca } \\
\text { p. cent }\end{array}$ & $\begin{array}{c}\mathrm{Mg} \\
\text { p. cent }\end{array}$ & $\begin{array}{l}\mathrm{H}_{2} \mathrm{O} \\
(100)\end{array}$ & $\begin{array}{c}\mathrm{N} \\
(100)\end{array}$ & $\begin{array}{l}\mathrm{P}_{2} \mathrm{O}_{5} \\
(100)\end{array}$ & $\underset{(100)}{\mathrm{K}}$ & $\begin{array}{c}\mathrm{Ca} \\
(100)\end{array}$ & $\begin{array}{c}\mathrm{Mg} \\
(100)\end{array}$ \\
\hline Robusta & $\begin{array}{l}68 \\
69\end{array}$ & $\begin{array}{l}7,9 \\
7,0\end{array}$ & $\begin{array}{l}1,12 \\
1,10\end{array}$ & $\begin{array}{l}33,5 \\
28\end{array}$ & $\begin{array}{l}6,11 \\
6,25\end{array}$ & $\begin{array}{l}171,6 \\
145,0\end{array}$ & $\begin{array}{l}1,62 \\
1,53\end{array}$ & $\begin{array}{l}0,24 \\
0,47\end{array}$ & $\begin{array}{l}1,25 \\
1,05\end{array}$ & $\begin{array}{l}4,10 \\
5,2\end{array}$ & $\begin{array}{l}0,35 \\
0,24\end{array}$ & $\begin{array}{l}57,5 \\
40,0\end{array}$ & $\begin{array}{l}0,54 \\
0,40\end{array}$ & $\begin{array}{l}0,08 \\
0,13\end{array}$ & $\begin{array}{l}0,42 \\
0,30\end{array}$ & $\begin{array}{l}1,38 \\
1,45\end{array}$ & $\begin{array}{l}0,12 \\
0,07\end{array}$ \\
\hline I-2 14 & $\begin{array}{l}68 \\
69\end{array}$ & $\begin{array}{l}8,6 \\
7,8\end{array}$ & $\begin{array}{l}1,15 \\
1,19\end{array}$ & $\begin{array}{l}50,0 \\
33\end{array}$ & $\begin{array}{l}7,68 \\
6,8\end{array}$ & $\begin{array}{l}135,8 \\
138,0\end{array}$ & $\begin{array}{l}1,48 \\
2,02\end{array}$ & $\begin{array}{l}0,23 \\
0,45\end{array}$ & $\begin{array}{l}1,22 \\
1,00\end{array}$ & $\begin{array}{l}2,58 \\
5,4\end{array}$ & $\begin{array}{l}0,24 \\
0,27\end{array}$ & $\begin{array}{l}69,0 \\
50,0\end{array}$ & $\begin{array}{l}0,74 \\
0,70\end{array}$ & $\begin{array}{l}0,11 \\
0,15\end{array}$ & $\begin{array}{l}0,61 \\
0,30\end{array}$ & $\begin{array}{l}1,29 \\
1,80\end{array}$ & $\begin{array}{l}0,12 \\
0,08\end{array}$ \\
\hline
\end{tabular}


De plus, ces feuilles hautes présentent de plus fortes concentrations en éléments nutritifs principaux ( $\mathrm{N}, \mathrm{P}$ et $\mathrm{K})$, ce qui permet d'espérer une meilleure précision des résultats analytiques.

Il semble donc, d'après ces considérations, que le niveau $\mathrm{H}$ ait des chances d'être le plus intéressant pour un diagnostic de nutrition. C'est la conclusion provisoire que nous adopterons, tout en restant conscient qu'elle doit être vérifiée, par une étude sur des essais de fertilisation existants, en prélevant aux trois niveaux sur des arbres ayant reçu des traitements différents, de façon à savoir quel est le niveau qui assure la meilleure discrimination entre des stations de fertilité différente (études en cours).

\section{2. - Influence de la période de prélèvement}

\subsection{Caractères physiques et morphologiques.}

D'après les figures 1 et 2, la croissance des feuilles ( $L$ et Ps) se prolonge pendant toute la saison de végétation, mais elle est maximale au milieu du cycle annuel. On remarque également que Ia décroît régulièrement pour les feuilles hautes. Ceci, bien que ce soit évidemment des feuilles différentes qui sont prélevées à chaque période, peut signifier une croissance continue des feuilles hautes.

Enfin, nous constatons que chez Robusta, l'indice de densité, (Id) présente un maximum en septembre, puis décroît significativement jusqu'à une sénescence des tissus avec migration de substances vers la tige juste avant la chute des feuilles qui a lieu en octobre chez Robusta. Ceci ne serait pas visible chez I-214 du fait de la défoliation plus tardive de ce clone.

\subsection{Teneurs relatives en eau et en éléments minéraux.}

A l'exception de $\mathrm{P}_{2} \mathrm{O}_{5}$ p. cent chez I-214, qui crôt jusqu'à la fin des observations, les teneurs en $\mathrm{N}, \mathrm{P}_{2} \mathrm{O}_{5}$ et $\mathrm{K}$ diminuent au printemps, puis augmentent jusqu'en septembre pour décroître avant la chute des feuilles. Ces trois stades peuvent être interprétés de la façon suivante :

- juste après le débourrement, les feuilles grandissent rapidement et sont riches en eau, mais les éléments minéraux N, P et K proviennent surtout des réserves de l'arbre et du bourgeon, en quantité limitée. Par effet de dilution, les teneurs relatives diminuent donc, feuilles,

- pendant l'été, l'arbre absorbe par ses racines, et les ions se concentrent dans les

-- avant la chute des feuilles, N, P et $\mathrm{K}$ migrent vers les rameaux pour reconstituer les réserves.

Les cations bivalents ont un comportement très différent : Ca présente un maximum en été, et $\mathrm{Mg}$ un minimum.

La teneur en eau décroît tout au long de la saison de végétation.

En première approximation, la validité du diagnostic foliaire imposerait donc de prélever les feuilles hautes en août ou début septembre pour les raisons suivantes :

- pendant cette période, la composition des feuilles varie peu. 
- au cours de la saison de végétation, l'écart entre la composition des feuilles hautes et des feuilles basses ne fait qu'augmenter. Conformément aux remarques faites dans le paragraphe 3-1 et qui tendaient à faire choisir le niveau supérieur comme plus représentatif de la nutrition de l'arbre, nous avons intérêt à prélever assez tard pour amplifier les caractères propres du niveau supérieur, mais avant les transformations précédant la chute des feuilles.

Si nous comparons nos résultats à ceux de Touzet, HeInrich et NoHN (déjà cités), nous constatons des différences. C'est donc avec juste raison que ces auteurs concluent que le diagnostic foliaire chez le peuplier doit être utilisé prudemment et mis au point pour chaque grande région climatique.

\subsection{Poids d'eau et d'éléments minéraux dans 100 feuilles.}

Tous ces caractères présentent une tendance générale à l'accroissement, ce qui traduit une accumulation de matières minérales dans le feuillage. Cependant, on note des exceptions :

- K (100) décroît brutalement chez les deux clones avant la chute des feuilles; il peut s'agir d'une migration de potassium vers la tige.

- $\mathrm{Ca}(100)$ et $\mathrm{Mg}$ (100) évoluent sensiblement comme Ca p. cent et $\mathrm{Mg}$ p. cent. La diminution de $\mathrm{Ca}$ p. cent et $\mathrm{Ca}$ (100) avant la chute des feuilles est à l'encontre de ce que l'on observe généralement chez d'autres essences où les feuilles s'enrichissent en calcium avant de tomber, opérant ainsi une sorte d'excrétion du calcium en excès. Peut-être devons-nous rapprocher cette particularité du peuplier du fait qu'il accumule des cristaux de carbonate de calcium dans son bois de cœur (CLÉMENT et JANIN 1972).

\section{3. - Influence du clone et niveau de nutrition}

Nous avons vu que l'influence du niveau et de la date de prélèvement se manifestent presque de la même façon sur les deux clones. Il n'en est pas de même en ce qui concerne le niveau de nutrition :

Le tableau 2 donne les valeurs des caractères observés le 8 septembre sur les feuilles hautes ainsi que les valeurs en éléments majeurs considérés comme optimales et relevées dans la bibliographie (Bonneau, 1968, Garbaye 1959, Leroy 1968).

On constate tout d'abord que les valeurs trouvées avoisinent celles que d'autres auteurs considèrent comme optimales. Ceci est en accord avec le sol riche et la croissance satisfaisante des arbres.

On remarque également que les teneurs sont nettement plus élevées chez I-214 que chez Robusta malgré la similitude de fertilité des sols; le calcium se comporte cependant différemment : ce même résultat a été acquis lors d'une étude antérieure portant sur plusieurs stations (GARBAYE 1969).

Ces observations entraînent une conclusion importante : le diagnostic foliaire doit s'appuyer sur des valeurs caractéristiques adaptées à chaque clone. Celles-ci devront être établies par des analyses dans des essais de fertilisation, sur des arbres recevant différents niveaux d'alimentation minérale. 


\section{4. - Caractères des feuilles de Robusta tombées à terre le 7 octobre}

Ces observations n'ont été faites que sur la station de Robusta, car ce clone perd des feuilles bien avant le I-214.

Il y avait du vent le jour du prélèvement et nous avons pris soin de ne ramasser que les feuilles fraîchement tombées, c'est-à-dire de couleur jaune et n'ayant subi encore ni dessèchement, ni lessivage, ni décomposition.

Comme il était impossible de connaître la provenance exacte de chaque feuille, nous n'avons constitué qu'un seul échantillon correspondant à l'ensemble de la station.

L'échantillon était d'ailleurs relativement homogène, car dépourvu des feuilles du niveau $\mathrm{H}$ qui étaient encore vertes et qui ne devaient tomber qu'une semaine plus tard.

Le tableau 3 donne les résultats de l'analyse de cet échantillon, ainsi que les valeurs moyennes correspondant aux feuilles prélevées sur les arbres aux trois niveaux habituels, à la même date.

Les valeurs des caractères morphologiques $\mathrm{L}$ et Ia (qui ne peuvent pas changer chez des feuilles sénescentes) situent les feuilles tombées comme provenant en moyenne du niveau $\mathbf{M}$ ou très légèrement au-dessus.

Il en est de même pour les autres caractères, à l'exception de l'azote et du phosphore qui sont moins abondants dans les feuilles tombées à terre et ont probablement migré vers les organes de réserve juste avant la chute.

En nous reportant au paragraphe 3.23, nous pouvons comparer le comportement du potassium, du phosphore et de l'azote :

- le potassium, élément qui n'est pas intégré dans les formes métaboliques, demeure très mobile dans les tissus; il migre vers la tige dès le début du mois de septembre, ce qui fait que les feuilles, même encore présentes sur l'arbre, sont appauvries en potassium.

- l'azote et le phosphore, liés aux combinaisons organiques, ne migrent vers la tige que beaucoup plus tard, immédiatement avant l'abscission et la chute des feuilles.

\section{5. - Comparaison des caractères foliaires entre deux années consécutives}

Le tableau 4 donne, pour les mêmes stations, les valeurs des caractères foliaires le 24 août 1968 et le 8 septembre 1969 pour les feuilles du bas.

On constate de nettes différences. Dans le cas des caractères chimiques, les valeurs sont plus élevées en 1968 qu'en 1969, à l'exception du phosphore $\left(\mathrm{P}_{2} \mathrm{O}_{5}\right.$ p. cent et $\mathrm{P}_{2} \mathrm{O}_{5}(100)$ et du calcium (Ca p. cent et Ca (100).

Il semble que l'on puisse attribuer ces différences à des facteurs climatiques plutôt qu'aux écarts entre dates de prélèvement (les précipitations ont été plus abondantes en 1968 qu'en 1969).

Par conséquent, il est imprudent, au cours d'une enquête, de comparer des analyses foliaires faites sur plusieurs années ou dans des zones climatiques différentes. 


\section{6. - Conclusion}

Le tiers supérieur de la couronne a un rôle prépondérant pour la quantité de matière emmagasinée dans les feuilles ainsi que pour les variations des teneurs des différents éléments minéraux pendant la saison de végétation. Par contre, les feuilles du reste de l'arbre montrent une variation plus faible tout au long de l'été.

Notre but étant de déterminer les conditions optimales de prélèvement pour une enquête sur la nutrition des peupleraies, nous avons choisi de prélever les feuilles du sommet de l'arbre comme étant les plus aptes à servir de base à un diagnostic foliaire.

Toutefois, lorsqu'on veut comparer les stations entre elles, il est nécessaire de choisir une période pendant laquelle la variation de composition des feuilles dans le temps est lente. La période qui convient le mieux de ce point de vue semble se situer fin août et début septembre.

Ces résultats provisoires demandent à être précisés par des prélèvements sur des arbres carencés ou au contraire à l'optimum de nutrition (étude en cours dans deux essais de fertilisation).

Nous remarquons également que les deux clones étudiés présentent des teneurs différentes en éléments nutritifs. Par conséquent, le diagnostic foliaire devra s'appuyer sur des valeurs caractéristiques propres à chaque clone.

Enfin, la comparaison des résultats des analyses foliaires faites en 1968 et 1969 sur les mêmes arbres et à la même période montrent de grandes différences vraisemblablement dues à des différences climatiques. Ceci, rapproché des résultats d'autres auteurs ayant travaillé dans une autre région, montre qu'il est impossible de comparer des valeurs obtenues sur plusieurs années ou dans des zones climatiques trop différentes.

Ou alors, comme le suggère Evers (1972), il convient de disposer de «stations-témoins » faisant l'objet d'analyses foliaires annuelles, ce qui permet de corriger les variations dues aux conditions climatiques propres à chaque année. Cette méthode présente également l'intérêt de pouvoir donner une idée juste du niveau réel de nutrition d'une peupleraie par rapport à un seuil de carence ou un optimum.

Reçu pour publication en septembre 1972.

\section{SUMMARY}

INFLUENCE OF DATE AND LEVEL OF SAMPLING ON LEAF ANALYSIS FOR TWO CLONES OF POPLAR

In order to determine the best way of sampling poplar leaves for foliar diagnostic, monthly sampling have been made from june to october, on three different levels, on a Robusta stand and an I - 214 stand, in the Marne Valley. The data are interpreted by statistical methods and the conclusions are as follows :

- Top-leaves are most interesting for foliar diagnostic because of their high concentration of macro-nutrients and because of their more intense metabolic activity which gives a better estimation of the nutritional state of the tree.

- Sampling must be done in august or in early september because leaf-content does not change much during this period. 
- The two clones have different leaf-contents. As a consequence, foliar diagnotic must be based on different values for optima or deficiency level.

- The comparison of data between 1968 and 1969 on the same trees shows great differences. So, during an investigation, it is impossible to compare foliar analysis made on different years or in regions climatically too different.

\section{ZUSAMMENFASSUNG}

\section{EINFLUSS DES DATUM UND DER HÖHE DEN PROBENENTNAHME}

\section{AUF DIE ERGEBNISSEN DER PAPPELBLATTANALYSEN}

Rostpappel und I-214 Pappel B1ättern wurden monatlich (von Juni bis Oktober) und an verschiedenen Baumhöhen in dem Marne Tal gesammelt und die beste Methode für Blattanalyse heraus zufinden.

Die Daten wurden statistischerweise ausgelegt und die wesenlichste Ergebnisse sind die folgenden :

- Die Blätter der obersten Wirteln sind am interessantesten für das Blätterdiagnostik, wegen ihrem Makronährstoffgehalt und ihrem grösserem Metabolismus die die Mineralernährungs möglichkeiten des Baumes besser anzeigen.

- Die Probenenentnahme muss man im August oder Anfang September durchfüren. Während dieser Periode, veränderen sich die Mineralstoffgehalten sehr wenig.

- Beiden Pappeln Klonen haben verschiedenen Mineralstoffgehalten. Deswegen muss sich das Blattdiagnostik auf verschiedenen kritischen werten stützen (Mangelgrentze und optimum).

- Das vergleichen der Daten von den selben Bäumen im Jahre 1968 mit den Daten im Jahre 1969 zeigt grosse Unterschieden in den Mineralernährstoffgehalten.

- Folglich ist es unmöglich die Blattanalysedaten von mehrere Jahren, oder von verschiedenen Klimazonen zu vergleichen.

\section{RÉFÉRENCES BIBLIOGRAPHIQUES}

(Anonyme) 1961. - The method of leaf sampling for chemical analysis. Korte Mededeling Bosbouwproefsta. Wageningen, $n^{\circ} 4(12)$.

Clément A, Janin G. 1971. - Mise en évidence des cristaux de carbonate de calcium dans le bois du genre populus. Conséquence sur la répartition des ions minéraux liés à la duraminisation. Ann. Sci. forest., 1972, 29, (1).

Bonneau M. 1968. - La fertilisation des forêts. B.T.I., 231.

BONNEAU M. 1968. - La fertisation des for éléments nutritifs dans les aiguilles d'Épicéa et leur signification pour l'interprétation des analyses d'aiguilles. Allgemeine Forest und Jagd Zeitung, 1972.

Fritzche K. - Beziehungen zwischen den Blattspiegelwerten, den Bodennährstoff gehalt und den Wachstum einiger Schwartz und Balsampappeln - Düngung und Melioration in der Forstwirtschaft. Deutsche Akademie der Landwirtschaftwissenschaften. Tagungsberichte $n^{\circ}$ 66, 169-192.

Garbaye J. 1969. - Le diagnostic foliaire chez le Peuplier. Note C.N.R.F. Nancy.

Garbaye J. 1969. - Comment aborder l'étude des facteurs de production du Peuplier en France - premiers résultats. Note C.N.R.F. Nancy.

Givlimondi G. 1960. - G. Ricerche preliminari sulla nutrizione minerale del pioppo a mezzo dell'analisi fogliare. Publ. centra. sper. agric. fer. 4, 231-245.

Leroy Ph., 1968. - Variations saisonnières des teneurs en eau et éléments minéraux des feuilles de chêne pédonculé. Ann. Sci. forest., 25, 2.

Schmalfuss K. Schulze W. 1961. - The accumulation of nutrients in tree species. Flora, Jena 150, 2-2.

STEFAN K. 1969. - The influence of sampling on the result of chemical foliar analysis - Working group of diseases. Commission Internationale du Peuplier F.A.O. Vienne.

Touzet G., Heinrich J.-C., NoHN I. - Diagnostic foliaire et vitesse de croissance. AFOCEL 1969.

Touzet G., HEINRICH J.-C., HernRich J.-C. 1970. - Concentration foliaire en azote, phosphore, potassium et calcium du Peuplier c.v. I. 214. AFOCEL, 1970.

VAn der Meiden, H.-A. 1959. - Studies on the importance of K to poplars. Kali, Amsterdam 4 (40) 371 376.

VAn der Meiden, H.-A., Kolster H.-W. 1964. - Variation de la composition minérale des feuilles de peuplier pendant la période de végétation dans différentes parties de l'arbre. Korte Mededelin $n^{\circ} 61$ Wageningen. 